\title{
Motivación y su influencia en el desempeño académico de los estudiantes de educación básica superior
}

\section{Motivation and its influence on the academic performance of students in basic higher education}

Motivación de los estudiantes

Audrey María Soledispa Rivera ${ }^{(1)}$

Evelyn Juliana San Andrés Soledispa ${ }^{(2)}$

Rafael Antonio Soledispa Pin ${ }^{(3)}$

(1) Unidad Educativa Emilio Bowen Roggiero. Manta. Ecuador. email: audreysoledispa5@gmail.com, ORCID: https://orcid,org/0000-0001-5707-2708.

(2) Unidad Educativa Emilio Bowen Roggiero. Manta. Ecuador. email: eve.jss2020@gmail.com, ORCID: https://orcid.org/0000-0003-2464-4663

(3) Universidad Laica Eloy Alfaro, Manta-Manabí-Ecuador. E-mail: rafaels947@hotmail.com, https://orcid.org/0000-0002-8058-1364

Contacto: audreysoledispa5@gmail.com

\section{Recibido: 9-10-2020 Aprobado: 30-11-2020}

\section{Resumen}

La motivación es de vital importancia para el aprendizaje de los educandos, porque constituye el punto de partir para aprender, crea el impulso de actuar y hace posible el aprendizaje. El presente tiene por objetivo determinar el grado de influencia de la motivación en el desempeño académico de los estudiantes. Se emplearon los métodos de investigación: empírico, descriptivo e investigativo, se aplicó una entrevista a la psicóloga de la institución y la recolección de datos se hizo mediante la técnica de la encuesta aplicada a 66 estudiantes. Los resultados de los instrumentos demostraron que a los estudiantes les falta determinar sus intereses y necesidades, no se han fijado metas, ni proyectos de vida. Es el docente y los padres de familias que tienen que ayudar a desarrollar las capacidades y 
habilidades con el fin de prepararlos para la vida y para adquirir el cambio de actitud del educando se debe aplicar una motivación eficaz en el proceso educativo; enseñar hábitos de estudios para que se interesen, valoren la educación y tomen conciencia de que ésta es la única que sacará del subdesarrollo a la sociedad.

Palabras Clave: Motivación, desempeño académico, enseñanza, aprendizaje, estudiante.

\begin{abstract}
Motivation is vitally important for learners' learning, because it is the starting point for learning, creates the drive to act, and makes learning possible. The objective of this document is to determine the degree of influence of motivation on the academic performance of students. The research methods were used: empirical, descriptive and investigative, an interview was applied to the psychologist of the institution and the data collection was done through the survey technique applied to 66 students. The results of the instruments showed that the students lack to determine their interests and needs, they have not set goals, or life projects. It is the teacher and the parents of families who have to help develop the capacities and abilities in order to prepare them for life and to acquire the change of attitude of the student, an effective motivation must be applied in the educational process; teach study habits so that they are interested, value education and become aware that this is the only one that will bring society out of underdevelopment.
\end{abstract}

Keywords: Motivation, academic performance, teaching, learning, student.

\title{
Introducción
}

En la actualidad es preocupante la falta de interés que demuestran los estudiantes de la Educación Básica superior, es por esta razón que se ha propuesto investigar sobre esta problemática que afecta a los jóvenes a nivel general y que les impide el desarrollo de destrezas y habilidades, uno de éstas es la falta de motivación de los educandos en el proceso educativo.

La educación debe promover el ejercicio activo de la individualidad del ser y ello solo se produce por la voluntad del querer, esa voluntad hay que estimularla para que mueva todo el proceso de aprendizaje. La motivación crea el impulso de actuar y hace posible la enseñanza aprendizaje del educando. 
Al hablar de motivación, consiste en analizar el estado de ánimo que manifiestan los estudiantes en su proceso enseñanza- aprendizaje, de allí que para que un estudiante rinda óptimamente, debe estar bien motivado. Este es un aspecto que aparece de manera recurrente al hablar de causas del desempeño académico, explica el inicio, dirección y perseverancia hacia un determinado objetivo (Usán \& Salavera, 2018). La motivación es el factor predominante para que el niño, adolescente se sienta a gusto en su proceso educativo; los docentes, padres de familias y estudiantes deben trabajar en conjunto para conseguir el éxito del mismo.

En el ámbito educativo, se define a la motivación como el interés que tiene el estudiante en aprender a aprehender, para así, crear su propio aprendizaje, aplicando actividades activas, dinámicas, y críticas, que le lleven a construir su conocimiento. El educando debe estar preparado para lo que demanda la educación del siglo XXI, basándose en el aprender a conocer, aprender a hacer, aprender a vivir juntos y aprender ser, para adquirir un aprendizaje significativo (Pico, 2017).

Para Pintrich y García, la motivación es una dimensión que se encuentra vinculada entre sí, formada por la motivación intrínseca o extrínseca, la valoración de las tareas, los sentimientos de autoeficacia, las creencias de control y la ansiedad que hace al educando un ser completo con actitud positiva para su aprendizaje (Gil \& Monroy, 2019).

La motivación según González Rey, radica en la subjetividad del individuo y representa la capacidad humana para crear sentidos subjetivos especiales, que simbolizan la forma emocional de toda destreza en el contexto del saber (Pérez \& Arcila, 2020). La motivación es un proceso psicológico que describe el nivel de interés, los motivos y necesidades que se presentan en el hombre.

Las habilidades motivadoras de los docentes han obtenido resultado positivos en los estudiantes, al aplicar estrategias de enseñanza-aprendizaje relevantes e innovadoras; y mejoran significativamente los resultados de aprendizaje, estas son la organización, comprensión, estrategias de apoyo, autoeficacia y sustentan la motivación en el aula (Barca, y otros, 2012).

En el aprendizaje de los educando el docente desempeña un lugar preponderante, porque es él quien influye en los saberes: conocer, hacer, ser y convivir, que adquieren y desarrollan los jóvenes para conseguir una educación integral, donde el estudiante esté preparado para competir en este mundo (Ramos \& Gómez, 2019). 
El desempeño académico no es otra cosa que la capacidad del estudiante de expresar lo aprendido y el esfuerzo puesto en su educación, el cual es evaluado de manera formal e informal. Este desempeño se refleja en diversas formas como es la capacidad de resolver problemas, desarrollo de habilidades y destrezas para competir en el mundo laboral, social y global (Sulgey, 1997)

El desempeño académico de los estudiantes está determinado por su potencial cognitivo, pero muchas veces ese potencial no se refleja en su interés académico y se establece la interrogante ¿por qué los jóvenes demuestran un desinterés en sus estudios?, llegando a la conclusión que el descuido y desinterés es por falta de motivación y esto lleva al educando a obtener bajas notas en su proceso educativo; podemos definir a la motivación como el conjunto de conocimientos implicados en la activación, dirección y persistencia de la conducta, el ser humano triunfa cuando es capaz de definir su propósito de vida, desarrolla su capacidad intelectual, afectiva y física y sobre todo difunde valores en los demás (Margarito, 2019)

La presente investigación tiene como objeto determinar el grado de influencia de la motivación en el desempeño académico de los estudiantes, parte fundamental para que ellos se sientan con ese interés de satisfacer sus necesidades y de alcanzar sus objetivos.

\section{Materiales y Métodos}

La investigación se realizó a los estudiantes de la Unidad educativa Emilio Bowen Roggiero de la ciudad de Manta, ubicada en la Parroquia Eloy Alfaro, donde se seleccionó a jóvenes de Décimo año de Educación General Básica del año lectivo 2020- 2021.

Los métodos utilizados fueron el empírico, descriptivo e investigativo, se realizó entrevista a la Psicóloga de la Institución Lic. Lorena Andrade para saber sobre el desempeño académico de los estudiantes.

Las autoras del presente trabajo son docentes de la Unidad educativa Emilio Bowen Roggiero y declaran no tener conflictos ni intereses en la investigación.

Se conversó con los estudiantes sobre el tema de la investigación y se les aplico una encuesta elaborada a través de un cuestionario web, donde 66 estudiantes de una población de 120 contestaron para obtener la base de datos y poder determinar si la motivación influye en el desempeño académico. 
Aspectos éticos a considerarse son la discrecionalidad de los datos, manejo ético de la información y la validación de la encuesta por expertos.

\section{Resultados.}

En la recolección de datos se obtuvieron resultados significativos y se analizó la influencia de la motivación en el desempeño académico de los estudiantes, tabla 1.

Tabla 1. Conocimientos sobre la necesidad de motivación para el aprendizaje

\begin{tabular}{|l|l|l|}
\hline Opciones & Frecuencia & Porcentaje \\
\hline Siempre & 52 & $78,80 \%$ \\
\hline A veces & 13 & $19,70 \%$ \\
\hline Nunca & 1 & $1,50 \%$ \\
\hline Total & 66 & $100 \%$ \\
\hline
\end{tabular}

Los estudiantes están conscientes de que para aprender tienen que estar motivados, ante lo cual, se exhorta a los docentes que mantengan siempre presente la motivación hacia el aprendizaje para lograr conocimientos, que le sirvan a los estudiantes participen activamente, se conviertan en investigadores para construir su propio aprendizaje, deben motivarse constantemente ellos mismos y así conquistar todo lo que se proponen, tabla 2.

\begin{tabular}{|l|l|l|}
\hline Opciones & Frecuencia & Porcentaje \\
\hline Media & 36 & $54,55 \%$ \\
\hline Alta & 28 & $42,42 \%$ \\
\hline Baja & 2 & $3,03 \%$ \\
\hline Total & 66 & $100 \%$ \\
\hline
\end{tabular}

Tabla 2. Grado de motivación de los estudiantes dentro del salón de clases

A los estudiantes les hace falta motivación dentro del salón de clase, ante lo cual, se invita al docente que debe crear un ambiente escolar agradable para que los jóvenes se sientan motivados y predispuestos a su enseñanza-aprendizaje. Las clases deben ser llamativas, didácticas y motivadoras. Para lograr una alta motivación se debe aplicar estrategias de enseñanza-aprendizaje relevantes e innovadoras; y mejoran significativamente los resultados de aprendizaje, estas son la organización, comprensión, estrategias de apoyo, autoeficacia y sustentan la motivación en el aula, tabla 3.

\begin{tabular}{|l|l|l|}
\hline Opciones & Frecuencia & Porcentaje \\
\hline Ciencia Naturales & 19 & $28,79 \%$ \\
\hline
\end{tabular}




\begin{tabular}{|l|l|l|}
\hline Ingles & 11 & $16,67 \%$ \\
\hline Estudios Sociales & 10 & $15,15 \%$ \\
\hline Matemática & 9 & $13,64 \%$ \\
\hline Educación Física & 9 & $13,64 \%$ \\
\hline Lengua y Literatura & 5 & $7,58 \%$ \\
\hline Educación Cultural y Artística & 3 & $4,55 \%$ \\
\hline Total & 66 & $100,00 \%$ \\
\hline
\end{tabular}

Tabla 3. Área de estudios en que se sienten motivados los estudiantes

Al aplicar la investigación a los estudiantes de décimo año de Educación General Básica Superior, revelan que en la mayoría de las áreas el maestro no efectúa una verdadera motivación. El docente solo imparte conocimientos y se olvida de la parte humana del estudiante, no se interesa por lo que le sucede al niño o adolescente, las clases son monótonas sin llamar la atención de los jóvenes. En las asignaturas que se sienten motivados es en Ciencias Naturales e inglés, tabla 4.

\begin{tabular}{|l|l|l|}
\hline Opciones & Frecuencia & Porcentaje \\
\hline Siempre & 38 & $57,58 \%$ \\
\hline A veces & 26 & $39,39 \%$ \\
\hline Nunca & 2 & $3,03 \%$ \\
\hline Total & 66 & $100 \%$ \\
\hline
\end{tabular}

Tabla 4. Conocimiento para saber si la motivación es permanente durante la hora de clases

Los estudiantes reconocen que los docentes utilizan la motivación al inicio y al final de clases, pero a pesar de aplicarla no consiguen el cambio de actitud de los adolescentes, les hace falta una verdadera motivación que les haga sentir lo importe que es prepararse para la vida y tener bien claro que la educación es el pilar fundamental para lograr lo que se proponen, tabla 5.

\begin{tabular}{|l|l|l|}
\hline Opciones & Frecuencia & Porcentaje \\
\hline Siempre & 8 & $12,12 \%$ \\
\hline A veces & 30 & $45,45 \%$ \\
\hline Nunca & 28 & $42,42 \%$ \\
\hline TOTAL & 66 & $100 \%$ \\
\hline
\end{tabular}

Tabla 5. La motivación que le dan los padres a sus hijos, cuando obtienen buenos resultados.

Son pocos los padres que incentivan a sus hijos dándoles un premio o regalo por sus notas alcanzadas, lo que demuestra que falta mayor atención. No es necesario recibir un regalo sino dar frases de aliento como ¡lo hiciste bien!, felicitarlo por sus notas para que así los estudiantes se sientan motivados a seguir adelante y alcanzar sus metas. La familia es un factor predominante para que el niño, joven se 
sienta motivado es por ello que los padres de familias deben estar siempre al lado de sus hijos demostrándole ese apoyo, interés y amor, tabla 6.

\begin{tabular}{|l|l|l|}
\hline Opciones & Frecuencia & Porcentaje \\
\hline Siempre & 54 & $81,82 \%$ \\
\hline A veces & 11 & $16,67 \%$ \\
\hline Nunca & 1 & $1,51 \%$ \\
\hline Total & 66 & $100 \%$ \\
\hline
\end{tabular}

Tabla 6. La motivación y su relación con el Proceso Enseñanza- Aprendizaje

Los estudiantes están conscientes de la relación que hay entre la motivación y el proceso enseñanza aprendizaje, por lo que se propone mantener esa vinculación para que el educando consiga desarrollar competencias y contenidos acorde al currículo educativo. No descuidar su enseñanza - aprendizaje para así conseguir adolescentes activos, dinámicos y productivos. El educando debe estar preparado para lo que demanda la educación del siglo XXI, basándose en el aprender a conocer, aprender a hacer, aprender a vivir juntos y aprender ser, para adquirir un aprendizaje significativo.

Estos resultados son de gran aporte para la investigación, porque proporciona una idea amplia de lo que opinan y sienten los estudiantes sobre la motivación que experimentan a diario, y cómo esta influye en su desempeño académico; además son significativos e invita a reflexionar sobre la labor que cumple el docente y la familia como apoyo para alcanzar objetivos en el proceso educativo.

\section{Discusión.}

La motivación es un proceso que se basa en la subjetividad del ser humano donde debe primar el interés que tiene la persona, los motivos y necesidades para lograr el éxito en la vida, si no están motivados no lograran alcanzar sus metas y objetivos, deben motivarse constantemente ellos mismos y así conquistar todo lo que se proponen (González, Mitjans Martínez, \& Bezerra, 2016), en los resultados obtenidos se evidencia que los estudiantes en un 78,80\% manifiestan es necesario estar motivados para aprender.

Al obtener los resultados de la investigación, ésta da a conocer que los estudiantes de Décimo año de Educación General Básica de la Unidad Educativa Emilio Bowen Roggiero, están conscientes de la relación que existe entre la motivación y el proceso enseñanza aprendizaje en un $81,82 \%$, es importante 
mantener esa vinculación para que el educando mantenga el interés por aprender y obtener mejores resultados en su desempeño académico, se concuerda con (Barca et al., 2012).

La motivación es estimular la pasión y el entusiasmo de las personas para contribuir con sus capacidades y competencias a la misión colectiva (Ortiz \& Agredal, 2018).Se define a la motivación como el entusiasmo para realizar sus habilidades y destrezas en el convivir diario. La motivación es considerada como la chispa que enciende al aprendizaje, su relación se representa como una doble vía, es decir que una depende de la otra, así también, es considerada el motor que impulsa al estudiante, a formar parte activa en el proceso de su desarrollo (Ospina, 2006).

La motivación es la clave para determinar el aprendizaje de los educando donde es necesario la relación con otras percepciones como el interés que tienen en cierto campo, la necesidad o privación de algo, valor sobre lo que se propone alcanzar y la aspiración, posibilidad de conseguir un logro. La correspondencia que existe entre motivación y desempeño académico se pone de manifiesto en el estudio realizado en la investigación, donde los educandos demuestran que les hace falta ese motor que los haga cambiar de actitud y estén predispuestos a su enseñanza aprendizaje (Rivera, 2013).

Los comportamientos observables que reflejan el nivel de motivación académica son, a su vez, de tres clases diferentes: hacer elecciones entre alternativas, estar activo e involucrado en las tareas y tener persistencia en ellas; el estudiante que se encuentra bien motivado puede desenvolverse en diferentes ámbitos dentro de su jornada de estudios (Rendón \& Coronel, 2019).

En el proceso educativo el docente debe considerar el tipo de estudiante a motivar, tomando en cuenta su personalidad, habilidades y destrezas, éste debe ser capaz de buscar metodologías acorde al estudiante para desarrollar estrategias que ayuden a fomentar la motivación intrínseca, donde aprendan a valorar el aprendizaje diario, disfruten lo aprendido y comprueben su progreso personal (Rinaudo, Chiecher, \& Donolo, 2003).

En los resultados los estudiantes manifestaron que solo en un 57,58\% los maestros utilizan la motivación durante todo la hora de clases, desde el inicio y el fin; para que el salón de clase se convierta en un clima agradable, el docente debe motivar, preparar temáticas de contenidos de aprendizaje llamativos, dar confianza a sus educandos, convertirse en amigos más que docente; para así 
favorecer el verdadero aprendizaje significativo de los estudiantes y debe estar presente durante todo el ciclo de aprendizaje (Pico, 2017; Polo \& Pingo, 2020).

La familia, base fundamental de la sociedad, es uno de los pilares para conseguir el proceso de la motivación, son ellos los primeros educadores de sus hijos en valores para obtener un autoestima muy elevado, es decir, que la motivación es a diario con una mirada de seguridad, con una frase de aliento, hace que los adolescentes se sientan bien y estén con ánimo de aprender y satisfacer sus motivos, intereses y necesidades.

Los resultados arrojaron que a veces en un 45,45\%, los educandos sienten la motivación por parte de sus padres, es decir que se debe trabajar con la familia, para que sean un ente de motivación para sus hijos, y así obtener mejores desempeños. La Psicología estudia las relaciones conjuntas entre los miembros de la familia, encaminadas al cumplimiento de las funciones familiares y los procesos de comunicación. (Domínguez \& García, 2017).

La misión de la educación es formar hombres para el presente y futuro, capaces de enfrentar los desafíos en su convivir diario, donde sea ejemplo de respeto, confianza y responsabilidad. Para alcanzar esta personalidad debe basarse en la formación humana que no es otra cosa que ser íntegro y la capacitación que es desarrollar habilidades y capacidades para desenvolverse en la era del conocimiento digital. El desempeño académico es la transformación que alcanzan los escolares en el aspecto cognitivo, actitudinal a través del proceso educativo que se demuestra en el modo como éstos se enfrentan en la vida y su relación con los demás (Quintero \& Orozco, 2013).

En el aprendizaje de los educando el docente desempeña un lugar preponderante, porque es él quien influye en los saberes: conocer, hacer, ser y convivir, que adquieren y desarrollan los jóvenes para conseguir una educación integral, esto se contrarresta con los resultados acerca de las materias en donde se sienten más motivados los estudiantes, y esto evidencia que hay la aplicación de diferentes grados de motivación y esto depende del maestro, de las técnicas, estrategias que aplique con sus estudiantes.

Los estudiantes del siglo XXI necesitan estar motivados para ejecutar sus propias ideas e intereses; estar en capacidad de realizar un aprendizaje autónomo, aprendizaje basado en proyecto, solución de problemas y así alcanzar el verdadero aprendizaje significativo, donde ellos tomen decisiones y estén 
listos para competir en el mundo globalizado. Es necesario que los estudiantes definan sus intereses, motivos y necesidades para sentirse bien y lograr un desempeño académico de excelencia como demanda el proceso educativo.

Se concuerdo con los estudios realizados por (González, Mitjans Martínez, \& Bezerra, 2016), aseveran que hace falta potenciar la motivación de los estudiantes donde ellos tengan claro cuáles son sus intereses, motivos y necesidades como lo propone el psicólogo Fernando González Rey se diferencia de las demás especies del planeta, quien puede cambiar su medio ambiente para que responda a sus propios intereses.

\section{Conclusión}

La motivación es vital en el desarrollo de la personalidad del ser humano, es el impulso para que el estudiante quiera alcanzar metas u objetivos, es por eso que se debe motivar cada momento a los estudiantes para que su desempeño académico sea óptimo. En la investigación se develó que los estudiantes necesitan estar motivados para llevar de mejor manera el proceso educativo y así obtener mejores resultados, esto es tarea de los docentes, estudiantes y padres de familia.

Al realizar esta investigación y tomando en cuenta los resultados arrojados, se observó que sí influye la motivación en el desempeño académico de los estudiantes de la Educación Básica Superior en el proceso de enseñanza-aprendizaje. Se considera pertinente que a mayor motivación se despierta el interés del estudiante por los conocimientos y habilidades a adquirir y depende del docente aplicar estrategias innovadoras y significativas para que el educando mantenga la atención en el proceso educativo y así mejorar el desempeño académico. Por otro lado, sería oportuno evaluar la motivación tomando en consideración los ambientes sociales más importantes del alumno, es decir dialogar si cómo se siente que es percibido en su casa, en la escuela y cómo se siente él mismo para detectar las fuentes extrínsecas que más influyen en su motivación.

Según los resultados obtenidos hay docentes que aplican mayor grado de motivación como es el caso de los docentes de Ciencias Naturales y Lengua extranjera; en el resto de los maestros es necesario un cambio o innovación para conseguir elevar los grados de motivación en sus estudiantes; convertirse en un orientador, guía, amigo de sus educandos para formar adolescentes predispuestos, activos, dinámicos y sobre todo con calidad humana. Para llegar a los estudiantes el docente debe motivar 
constantemente dentro y fuera del salón de clases, aplicar estrategias metodológicas activas para su inter - aprendizaje y así el adolescente desarrolle habilidades, destrezas y competencias que le sirvan para su desempeño competitivo en su vida profesional.

\section{Bibliografía}

Barca, A., Almeida, L., Porto, A., Peralbo, Manuel, \& Brenlla, J. (2012). Motivación escolar y rendimiento: impacto de metas académicas, de estrategias de aprendizaje y autoeficacia. Anales de Psicologia/Annals of Psychology, 28(3), 848-859. Recuperado de: https://revistas.um.es/analesps/article/view/analesps.28.3.156101/150191

Domínguez, R., \& García, D. (2017). La familia como grupo psicosocial y su vÍnculo con la escuela. Comité Editorial, 10(1), 354.

Gil, M., \& Monroy, M. (2019). Tecnología y motivación para el desempeño académico de alumnos en educación básica. Nuevos paradigmas en los procesos de enseñanza-aprendizaje, 66-74.

González, F., Mitjans Martínez, A., \& Bezerra, M. (2016). Psicología en la educación: Implicaciones de la subjetividad en una perspectiva Cultural-Histórica. Revista puertorriqueña de Psicología, 27(2), 260-274.

Margarito, G. (2019). Motivación y éxito en el rendimiento académico de los estudiantes del instituto de educación superior pedagógico privado Juan Pablo II. Huaral.

Ortiz, A. M., \& Agredal, M. (2018). Gamificación en educación: una panorámica sobre el estado de la cuestión. Educação e Pesquisa, 44.

Ospina, J. (2006). La motivación, motor del aprendizaje. Rev. Cienc. Salud. Bogotá, 4, 158-160.

Pérez, R., \& Arcila, P. (2020). IMPLICACIONES DE LA TEORIA DE LA SUBJETIVIDAD EN EL PROCESO DE ENSEÑANZA-APRENDIZAJE ESCOLAR.

Pico, M. (2017). La importancia de la motivación en el rendimiento académico de los estudiantes de secundaria 
https://dspace.uib.es/xmlui/bitstream/handle/11201/3589/Pico_Lozano_Marta.pdfsequence=1\&isA llowed $=\mathrm{y}$

Polo, F., \& Pingo, G. (2020). MOTIVACIÓN Y DESEMPEÑO ACADÉMICO DE ESTUDIANTES DE ADMINISTRACIÓN. YACHAQ, 2(2), 11-11.

Quintero, M., \& Orozco, G. (2013). El desempeño académico: una opción para la cualificación de las instituciones educativas. Plumilla Educativa, 12(2), 93-115. Obtenido de: https://dialnet.unirioja.es/servlet/articulo?codigo $=4756664$

Ramos, R., \& Gómez, N. (2019). La influencia del docente sobre la motivación, las estrategias de aprendizaje, pensamiento crítico de los estudiantes y rendimiento académico en el área de Educación Física. Psychology, Society, \& Education, 11(1), 137.

Rendón, M., \& Coronel, M. (2019). Tecnología y motivación para el desempeño académico de alumnos en educación básica. Nuevos paradigmas en los procesos de enseñanza-aprendizaje, 6674. Obtenido de: http://www.adayapress.com/wp-content/uploads/2019/05/Parad8.pdf

Rinaudo, M., Chiecher, A., \& Donolo, D. (2003). Motivación y uso de estrategias en estudiantes universitarios. anales de psicología, 19(1), 107-119. Obtenido de: https://revistas.um.es/analesps/article/view/27901/27031

Rivera, G. (2013). La motivación del alumno y su relación con el rendimiento académico en los estudiantes de Bachillerato Técnico en Salud Comunitaria del Instituto República Federal de México de Comayagüela. México.

Sulgey, D. S. (1997). Estudio de la posible relación entre autoestima, autodirección, motivación en el desempeño académico de alumnos de secundaria de una institución pública del municipio de Funza. Cundinamarca, , Colombia.

Usán, P., \& Salavera, C. (2018). Motivación escolar, inteligencia emocional y rendimiento académico en estudiantes de educación secundaria obligatoria. Actualidades en Psicología, 32(125), 95-112. 